\title{
Standard and non-standard primordial neutrinos
}

\author{
P. D. Serpico \\ Max-Planck-Institut für Physik, (Werner-Heisenberg-Institut), \\ Föhringer Ring 6, 80805, Munich, Germany \\ E-mail: serpico@mppmu.mpg.de
}

\begin{abstract}
The standard cosmological model predicts the existence of a cosmic neutrino background $(\mathrm{C} \nu \mathrm{B})$ with a present density of about $n_{\nu}+n_{\bar{\nu}} \simeq 110 \mathrm{~cm}^{-3}$ per flavour, which affects big-bang nucleosynthesis (BBN), cosmic microwave background (CMB) anisotropies, and the evolution of large scale structures (LSS). We report on a precision calculation of the $\mathrm{C} \nu \mathrm{B}$ properties including the modification introduced by neutrino oscillations. The role of a possible neutrino-antineutrino asymmetry and the impact of non-standard neutrino-electron interactions on the $\mathrm{C} \nu \mathrm{B}$ are also briefly discussed.
\end{abstract}

PACS numbers: 14.60.Lm, 98.80.-k

Keywords: neutrinos, cosmology

\section{Neutrino decoupling including oscillations}

In the early universe, thermally produced neutrinos were in equilibrium with other particles down to temperatures of few $\mathrm{MeV}$, when weak interactions became ineffective and neutrino decoupled from the plasma (for general and updated reviews on the $\mathrm{C} \nu \mathrm{B}$ and its impact on cosmology, the reader is addressed to [1, 2] ). As a first approximation, the thermal Fermi-Dirac momentum spectrum is preserved after the freeze-out of weak interactions, since neutrinos decoupled when ultra-relativistic and both neutrino momenta and temperature redshift identically with the universe expansion. Shortly after neutrino decoupling, the photon temperature dropped below the electron mass, and the $e^{ \pm}$annihilations heated the photons, whose temperature was thus raised with respect to neutrino one by a factor $z_{\text {fin }}^{0} \equiv(11 / 4)^{1 / 3} \simeq 1.4010$ in the limit of instantaneous neutrino decoupling. However, neutrino decoupling and $e^{ \pm}$annihilations were sufficiently close in time that some relic interactions between $e^{ \pm}$and neutrinos existed, especially in the UV tail of the distributions. One expects then a slightly smaller increase of the comoving photon temperature and non-thermal distortions in the neutrino spectra, larger for $\nu_{e}$ and $\bar{\nu}_{e}$, which also undergo charged current interactions with $e^{ \pm}$. In [3], we studied this process in detail, by solving numerically the momentum-dependent density matrix equations for the neutrino spectra, including finite temperature QED corrections to the 


\begin{tabular}{lcccccc}
\hline Case & $z_{\text {fin }}$ & $\delta_{e}(\%)$ & $\delta_{\mu}(\%)$ & $\delta_{\tau}(\%)$ & $N_{\text {eff }}$ & $\Delta Y_{p}$ \\
\hline No mixing, no QED & 1.3990 & 0.95 & 0.43 & 0.43 & 3.035 & $1.47 \times 10^{-4}$ \\
No mixing & 1.3978 & 0.94 & 0.43 & 0.43 & 3.046 & $1.71 \times 10^{-4}$ \\
$\left\{\theta_{12}, \theta_{23}, \theta_{13}\right\}=33^{\circ}, 45^{\circ}, 0^{\circ}$ & 1.3978 & 0.73 & 0.52 & 0.52 & 3.046 & $2.07 \times 10^{-4}$ \\
$\left\{\theta_{12}, \theta_{23}, \theta_{13}\right\}=33^{\circ}, 45^{\circ}, 12.5^{\circ}$ & 1.3978 & 0.70 & 0.56 & 0.52 & 3.046 & $2.12 \times 10^{-4}$ \\
$\left\{\theta_{12}, \theta_{23}, \theta_{13}\right\}=45^{\circ}, 45^{\circ}, 0^{\circ}$ & 1.3978 & 0.69 & 0.54 & 0.54 & 3.045 & $2.13 \times 10^{-4}$ \\
\hline
\end{tabular}

Table 1. Frozen values of the photon to neutrino "temperature ratio" $z_{\text {fin }}$, the energy density corrections $\delta_{\alpha}$, the parameter $N_{\text {eff }}$ and the change in the ${ }^{4} \mathrm{He}$ mass fraction $\Delta Y_{p}$ for different cases.

electromagnetic plasma, and for the first time in an exact way the effect of three-neutrino flavour oscillations.

The global contribution of neutrinos to the energy density is usually parameterized via an effective number of neutrinos $N_{\text {eff }}$ (=3 for instantaneous decoupling),

$$
N_{\text {eff }} \equiv \frac{\rho_{\nu+x}}{\rho_{\nu}^{0}} \frac{\rho_{\gamma}^{0}}{\rho_{\gamma}}=\left(3+\sum_{\alpha} \delta_{\alpha}\right)\left(\frac{z_{0}}{z}\right)^{4}
$$

where $\rho_{\gamma}^{0}$ and $\rho_{\nu}^{0}$ are respectively the energy density of the photon plasma and of a single neutrino species in the limit of instantaneous decoupling, $\rho_{\gamma}$ the actual energy content of the photon plasma, and $\rho_{\nu+x}$ the total energy content of weakly interacting particles (including possible exotic contributions). The second equality follows when only the three active neutrinos contribute to $\rho_{\nu+x}$; there, the different photon temperature evolution accounts for the second factor and the relative energy-density distortion in the $\alpha$-th neutrino flavour is given by $\delta_{\alpha} \equiv\left(\rho_{\nu_{\alpha}}-\rho_{\nu}^{0}\right) / \rho_{\nu}^{0}$. In Tab. 1 we report our results for neutrino distortion observables for several cases: the one without oscillations nor QED corrections to the plasma, the one adding only QED corrections, and three cases including oscillations, with the first one close to present best fit of neutrino world data. We find that, differently from QED effects, oscillations do not essentially modify the total change in the neutrino energy density, while the small effect on the production of primordial ${ }^{4} \mathrm{He}$ is increased by about $20 \%$ : BBN is indeed sensitive separately to $\delta_{e}$ and $N_{\text {eff }}$, and removes the degeneracy. The results are stable within the presently favoured region of neutrino mixing parameters. Note that from the distortions in the $\mathrm{C} \nu \mathrm{B}$ one would expect $\mathcal{O}(1 \%)$ change in the predicted helium fraction $Y_{p}$. However, the neutrino spectral distortions and the modified photon temperature evolution conspire to almost cancel each other, and $\Delta Y_{p} / Y_{p} \simeq \mathcal{O}(0.1 \%)$ only. Nonetheless, this effect is of the same order of the predicted uncertainty coming from the error on the measured neutron lifetime and has to be included in precise BBN predictions (see e.g. 4]).

\section{Neutrino asymmetry}

In the previous considerations we have neglected possible (comoving) neutrino chemical potentials $\xi_{\alpha}$, that would exist in the presence of a neutrino-antineutrino asymmetry. 
Is that a justified approximation? Primordial nucleosynthesis yields are sensitive to neutrino asymmetries via: $a$ ) the isospin-changing weak rates (a positive $\xi_{e}$ enhances $n \rightarrow p$ processes with respect to the inverse ones); $b$ ) a modified expansion rate, since non-zero $\xi_{\alpha}$ 's contribute to total $\mathrm{N}_{\text {eff }}$ as

$$
N_{\mathrm{eff}}\left(\xi_{\alpha}\right) \simeq N_{\mathrm{eff}}(0)+\sum_{\beta}\left[\frac{30}{7}\left(\frac{\xi_{\beta}}{\pi}\right)^{2}+\frac{15}{7}\left(\frac{\xi_{\beta}}{\pi}\right)^{4}\right] .
$$

The former effect is dominant, in particular on $Y_{p}$, and a stringent BBN bound on $\xi_{e}$ is obtained. Additionally, it was shown in [5] that neutrino oscillations lead to flavour equilibrium before BBN: If neutrino chemical potentials are non-vanishing, they should be equal to each other, and the BBN bound on $\xi_{e}$ extends to all flavours. If no exotic degrees of freedom are allowed in $N_{\text {eff }}(0)$ and the CMB prior on the baryon abundance is used, present constraints are at the level $-0.05<\xi_{e}<0.07$ [ 6 . It was however noted that if both $N_{\text {eff }}(0)$ and $\xi_{e}$ are allowed to vary, the BBN bounds on $N_{\text {eff }}$ and $\xi_{e}$ are somewhat relaxed [7, 8]. Still $\left|\xi_{e}\right| \lesssim 0.3$ holds, so that previous considerations and CMB and LSS observables can not be significantly affected by a $\nu-\bar{\nu}$ asymmetry, at least within present accuracy of cosmological observations.

\section{Non-standard physics \& neutrino decoupling}

If flavour-changing or non-universal coupling to electrons are present, they might delay the decoupling phenomenon: neutrinos get more energy from $e^{ \pm}$annihilations. Although standard non-thermal features are too small to be detected with present data, larger non-thermal distortions could be more easily detected. Phenomenologically, we can add to the standard model Lagrangian the effective operators

$$
\mathcal{L}_{\mathrm{NSI}}^{\alpha \beta}=-2 \sqrt{2} G_{F} \sum_{P} \epsilon_{\alpha \beta}^{P}\left(\bar{\nu}_{\alpha} \gamma^{\mu} L \nu_{\beta}\right)\left(\bar{e} \gamma_{\mu} P e\right)
$$

where $P=L, R=\left(1 \mp \gamma_{5}\right) / 2$ and the dimensionless $\epsilon_{\alpha \beta}^{P}$ parameterize the strength of non-standard interactions (NSI). For example, preliminary calculations show that, for

$\epsilon_{\tau \tau}^{L} \simeq \epsilon_{\tau \tau}^{R} \simeq 2, N_{\text {eff }}$ as large as 3.17 and a variation in the ${ }^{4}$ He yield almost of the order of $10^{-3}$ can be obtained. When limiting the NSI to vary within present laboratory bounds, one finds in general enhanced non-thermal features, with a value of $\left(N_{\text {eff }}-3\right)$ changed by up to a factor $\sim 3$ with respect to the standard case. The effect of NSI on the neutrino decoupling has been investigated in detail and the results have been reported in 9 .

\section{Conclusions}

Presently, $\mathrm{C} \nu \mathrm{B}$ is starting being tested at a non-trivial level by CMB and LSS data (see e.g. [10]), a progress which is nicely reflected in the sharpening of the bounds on $N_{\text {eff }}$ : although concerns still remain on possible unaccounted systematics, it is intriguing that for the first time the constraints quoted in 11, 12] are strong enough-e.g. comparable with the traditional bounds from $\mathrm{BBN}$ - to start discriminating among models. The 
PLANCK satellite temperature and polarization data alone will probably pin-down this observable to the level of $\Delta N_{\text {eff }}=0.2$, and future missions might reach the sensitivity of 0.04-0.05 needed to test the standard scenario [13, 14, 15, possibly ruling out many exotic models. Although no direct detection is possible, we expect a bright future for the $\mathrm{C} \nu \mathrm{B}$ !

\section{Acknowledgments}

I thank the organizers of SNOW 2006 for the very pleasant and stimulating workshop. It is a pleasure to thank G. Mangano, G. Miele, S. Pastor, T. Pinto, O. Pisanti, and G. Raffelt for all discussions and fruitful collaboration on the topics summarized in this paper.

\section{References}

[1] S. Hannestad, hep-ph/0602058.

[2] J. Lesgourgues and S. Pastor, Phys. Rept. 429, 307 (2006).

[3] G. Mangano et al, Nucl. Phys. B 729, 221 (2005).

[4] P. Serpico et al, JCAP 0412010 (2004).

[5] A. D. Dolgov et al , Nucl. Phys. B 632, 363 (2002).

[6] P. Serpico \& G. Raffelt, Phys. Rev. D 71, 127301 (2005).

[7] V. Barger et al , Phys. Lett. B 569, 123 (2003).

[8] A. Cuoco et al, Int. J. Mod. Phys. A 19, 4431 (2004).

[9] G. Mangano et al, hep-ph/0607267.

[10] R. Trotta \& A. Melchiorri, Phys. Rev. Lett. 95, 011305 (2005).

[11] U. Seljak, A. Slosar \& P. McDonald, astro-ph/0604335

[12] D. N. Spergel et al, astro-ph/0603449.

[13] R.E. Lopez et al , Phys. Rev. Lett. 82 (1999) 3952.

[14] R. Bowen et al, MNRAS 334 (2002) 760.

[15] S. Bashinsky \& U. Seljak, Phys. Rev. D 69 (2004) 083002. 